\title{
Licuefacción Solvotérmica de Madera de Acacia (Acacia mangium)
}

\author{
Alexander Osorio, Elkin Gómez, David Ocampo y Luis A. Rios*. \\ Procesos Químicos Industriales, Departamento de Ingeniería Química, Facultad de Ingeniería, Universidad \\ de Antioquia UdeA, Calle 70 No.52-21, Medellín, Colombia. (e-mail: alexander.osoriog@udea.edu.co; \\ david.ocampoe@udea.edu.co; elkina.gomez@udea.edu.co; luis.rios@udea.edu.co)
}

${ }^{*}$ Autor a quien debe ser dirigida la correspondencia.

Recibido Jun. 15, 2018; Aceptado Ago. 17, 2018; Versión final Sep. 14, 2018, Publicado Abr. 2019

\begin{abstract}
Resumen
Se presentan los resultados de la licuefacción solvotérmica de Acacia mangium. En primer lugar, se optimizaron temperatura, tiempo de reacción y relación solvente (agua)/biomasa mediante un diseño factorial $3^{k}$, encontrando que, a temperatura de $270^{\circ} \mathrm{C}$, una relación solvente (agua)/biomasa de 20 y tiempo de reacción de 60 minutos se obtiene el más alto rendimiento $(40,2 \%)$, con un poder calorífico de 29,63 $\mathrm{MJ} / \mathrm{kg}$. Al evaluar rendimiento energético, tamaño de equipos y consumo energético en la reacción, se obtiene que las mejores condiciones son $330^{\circ} \mathrm{C}$, una relación solvente (agua)/biomasa de 15 y un tiempo de reacción de 0 minutos, obteniéndose un biocrudo con rendimiento másico del $36 \%$ y poder calorífico de 31,7 $\mathrm{MJ} / \mathrm{kg}$. Se evaluaron otros solventes y se encontró que con acetona se obtienen rendimientos de hasta 47,8 $\%$, manteniendo estable el poder calorífico. El uso de atmósfera de hidrógeno solo tuvo efecto positivo usando acetona como solvente.
\end{abstract}

Palabras clave: biocombustibles; biocrudo; biomasa; Acacia mangium; licuefacción.

\section{Solvothermal Liquefaction of Acacia Wood (Acacia mangium)}

\begin{abstract}
Solvothermal liquefaction of Acacia mangium is presented. First, process variables, such as temperature, reaction time, and solvent-biomass ratio were optimized through $3^{\mathrm{k}}$ factorial experimental design. Results showed that at $270^{\circ} \mathrm{C}$ and solvent (water)-biomass ratio of 20- and 60-min reaction time, the highest biooil yield $(40,2 \%)$ and highest $\mathrm{HHV}$ value $(29,63 \mathrm{MJ} / \mathrm{kg}$ ) were obtained. When energy yield, equipment dimension and energy consumption were taken into account, the best experimental conditions are $330^{\circ} \mathrm{C}$ for the solvent temperature, solvent (water)-biomass ratio of 15 and 0 min reaction time, with biooil yield $36 \%$ and heat power of $31,7 \mathrm{MJ} / \mathrm{Kg}$. Higher biooil yields $(47,8 \%)$ resulted using acetone as solvent with almost the same heat power. Hydrogen atmosphere has a positive effect in the liquefaction only with acetone as solvent.
\end{abstract}

Keywords: biooil, biofuels, solvothermal liquefaction, Acacia mangium, biomass. 


\section{INTRODUCCIÓN}

Según la Reforestadora Integral de Antioquia, uno de los residuos forestales más importantes en la región Caribe y Alto Magdalena de Colombia, especialmente en Antioquia, es la Acacia (Acacia mangium), la cual es utilizada en fabricación de muebles y artículos de decoración (RIA, 2013). Colombia cuenta con 25 millones de hectáreas con aptitud forestal, en las cuales se planea en los próximos años, introducir especies con alta capacidad de producir madera con fines comerciales como teca (Tectona grandis L.F.), melina (Gmelina arborea Roxb.) y acacia (Acacia mangium Willd) (Espitia, 2010). Para el año 2014 el departamento de Antioquia ya contaba con 2100 hectáreas de Acacia, cantidad que se ha prácticamente duplicado en la actualidad. En el mundo, es una especie muy común en países como Filipinas, Malasia, Haití, India, Tailandia, Indonesia y Panamá. Gracias a condiciones agroecológicas, en Colombia existen varias especies de Acacia que registran rendimientos superiores a nuestros competidores alcanzando alrededor de $30 \mathrm{~m}^{3} / \mathrm{ha} / \mathrm{año}$, mientras los demás países reportan rendimientos entre $8-19 \mathrm{~m}^{3} / \mathrm{ha} / \mathrm{año}$ (Proexport, 2012).

Un estudio realizado por la UPME (Unidad de Planeación Minero Energética-Colombia) en el 2010 estableció que, por cada árbol utilizado para la producción maderera, solo se aprovecha comercialmente un $20 \%$, un $40 \%$ se deja en el campo en forma de hojas y ramas y el $40 \%$ restante en forma de aserrín, astillas y corteza (UPME Unidad de Planeación Minero Energética, 2010). Por este motivo, la UPME declaró a la especie forestal Acacia como un residuo energético potencial, ya que cerca del $80 \%$ podría ser transformado en combustibles renovables. Debido a su bajo costo y gran disponibilidad, la biomasa lignocelulósica, es una de las materias primas más promisorias para la generación de biocombustibles y precursores químicos a nivel mundial (Klass, 1998; Lloyd y Wyman, 2005), y tecnología de la licuefacción hidrotérmica es una de las más atractivas para este fin. Este proceso se puede definir como la transformación química de la biomasa, la cual es fragmentada en cadenas cortas de hidrocarburos, al ser sometida a alta presión y temperatura, en presencia de un solvente como el agua u otro líquido en estado supercrítico (Kersten et al. 2011; Vardon et al. 2012).

Diferentes autores han estudiado la licuefacción hidrotérmica de biomasas maderables. (Zhong y Wei 2004) estudiaron el efecto de la temperatura entre $280-360^{\circ} \mathrm{C}$ en la licuefacción hidrotérmica para las cuatro biomasas Cunninghamia lanceolata, P.massoniana, P. tomentosa Carr y Fraxinus Mandshurica, encontrando rendimientos con respecto a la biomasa alimentada entre 27,38 y $32,21 \%$, el cual aumenta con la temperatura; además reportaron que el uso de $\mathrm{K}_{2} \mathrm{CO}_{3}$ como catalizador disminuye los subproductos y aumenta el rendimiento a biocrudo. Otros autores (Liu y Zhang 2008) encontraron que el rendimiento a partir de madera de pino en presencia de varios solventes (aguas, acetona y etanol) mejora cuando se incrementa la temperatura cerca de los $300^{\circ} \mathrm{C}$ para el agua y $400^{\circ} \mathrm{C}$ para la acetona y el etanol. (Cheng et al. 2010) encontraron que disminuir la relación biomasa solvente incrementa el rendimiento a biocrudo y también la formación de residuos sólidos usando como solvente el agua en la licuefacción hidrotérmica (HTL) de aserrín de pino.

En el presente artículo se determinaron las mejores condiciones para la producción de biocrudo mediante la licuefacción solvotérmica de Acacia mangium, con el fin de obtener mayor rendimiento, alto poder calorífico y bajos puntos de destilación, modificando las variables de operación temperatura, relación biomasa/solvente, tiempo de reacción, carga de catalizador, tipo de solvente (agua, acetona, etanol y metanol) y atmosfera de reacción. El empleo de estos solventes en combinación con una atmosfera reductora no ha sido reportado y se constituye en un importante aporte tecnológico debido a las buenas propiedades del biocrudo obtenido.

\section{METODOLOGÍA}

La metodología se divide en cuatro subsecciones: materiales, experimentación, separación de los productos de reacción y caracterización de productos.

\section{Materiales}

La biomasa utilizada en la evaluación de la licuefacción hidrotérmica fue la Acacia mangium, suministrada por el RIA (Reforestadora Integral de Antioquia). Ésta fue recolectada de todas las zonas de cultivo del departamento de Antioquia, Colombia. El material fue sometido a un proceso de secado a $60^{\circ} \mathrm{C}$ hasta lograr una humedad inferior al 10\% para evitar su degradación biológica para su almacenamiento. También se realizó un proceso de conminución mecánica en un molino vegetal de cuchillas y tamizaje con el fin de homogenizar su distribución de tamaño de partícula [0,8-14 mm]. 


\section{Experimentación}

Se desarrolló un diseño experimental factorial $3^{k}$ con el fin de evaluar las variables temperatura, relación biomasa solvente y tiempo de reacción. Como catalizador se utilizó el carbonato de potasio $\mathrm{K}_{2} \mathrm{CO}_{3}$ (Mazaheri et al., 2013); (Xu y Lancaster, 2008), y agua como solvente inicial; luego se evaluó el efecto solvente de otras sustancias como acetona, etanol y metanol, y por último el efecto de una atmosfera reductora. En la tabla 1 se muestran las condiciones experimentales evaluadas para la licuefacción de Acacia mangium, las cuales se establecen basados en el estudio de varios autores como (Demirbas, 2008; Zhou et al., 2010; Anastasakia y Ross, 2011; Zhang et al., 2013; Dimitriadis y Bezergianni, 2017).

Tabla 1: Condiciones experimentales de la licuefacción hidrotérmica de las biomasas de referencia empleadas.

\begin{tabular}{ll}
\multicolumn{1}{c}{ Variable } & \multicolumn{1}{c}{ Valor } \\
\hline Presión inicial & $10 \mathrm{bar}$ \\
Temperatura & $270,300,330 \stackrel{\circ}{\mathrm{C}}$ \\
Tiempo & $0,30,60$ minutos \\
Tamaño de partícula & $0,8-1,4 \mathrm{~mm}$ \\
Solvente & Agua \\
Catalizador & $\mathrm{K}_{2} \mathrm{CO}_{3}$ \\
Carga de catalizador & $10 \%(\mathrm{p} / \mathrm{p})$ respecto a la biomasa \\
Atmósfera & Argón \\
Cantidad de biomasa & $6 \mathrm{~g}$ \\
Relación biomasa/solvente & $1: 10,1: 15,1: 20(\mathrm{~g} / \mathrm{g})$ \\
\hline
\end{tabular}

La licuefacción de biomasa se llevó a cabo en un reactor PARR4576 B de $250 \mathrm{~mL}$, el cual fue cargado con la biomasa, el solvente y el catalizador. Posteriormente, se presurizó con argón hasta una presión de 10 bares y se inició el calentamiento hasta la temperatura deseada con una agitación de 360 rpm, una vez alcanzada la temperatura de reacción se empezó a contar el tiempo de reacción; después del cual, el reactor se dejó enfriar hasta temperatura ambiente y se procedió a la separación de los productos de reacción.

\section{Separación de los productos de reacción}

Después de enfriar el reactor, se procedió a realizar la separación de productos y subproductos de reacción. La fracción gaseosa producida durante la reacción de licuefacción fue retirada del reactor al alcanzar la temperatura ambiente. El residuo sólido de la reacción se lavó con acetona para recuperar el biocrudo producido y se extrajo el biocrudo de la fase acuosa empleando diclorometano (Garcia-Perez et al. 2007; Oasmaa et al. 2003; Yang et al. n.d.; Zou et al. 2009). El diagrama de la Figura 1, muestra el procedimiento empleado en la separación de productos de reacción.

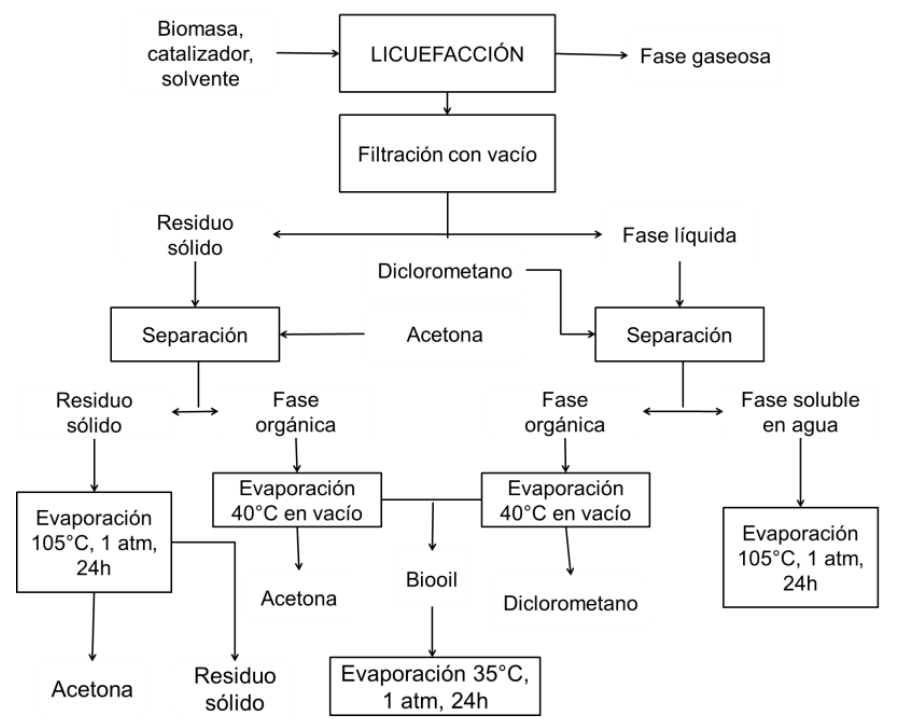

Fig. 1: Procedimiento para la separación de productos y sub-productos de reacción. 
Una vez separadas las tres fases: acuosa, biocrudo y sólida, se secaron para su posterior cuantificación y caracterización. Para calcular los rendimientos hacia las diferentes fracciones se emplearon las siguientes ecuaciones, teniendo en cuenta el contenido de materia orgánica presente en la biomasa inicialmente alimentada. Para estas ecuaciones se empleó el peso de los productos y subproductos secos.

$\%$ Rendimiento biocrudo $=\frac{\text { peso biocrudo }}{\text { peso biomasa }} \times 100$

$\%$ Rendimiento residuo sólido $=\frac{\text { peso fracción sólida }}{\text { peso biomasa }} X 100$

$\%$ Rendimiento biocrudo soluble en agua $=\frac{\text { peso fracción acuosa }}{\text { peso biomasa }} X 100$

$\%$ Rendimiento gases $=100-$ suma de rendimientos anteriores

\section{Caracterización de Productos}

Las composiciones elementales del biocrudo fueron determinadas usando un analizador elemental TruSepc CHNS-O micro marca Leco. El poder calorífico superior del biocrudo fue determinado usando la fórmula de Dulong (ecuación 5) (Zhuang et al. 2012; Duan et al. 2012; Duan et al. 2013; Duan y Savage 2011; Yuan et al. 2009). Además, el biocrudo obtenido después de la reacción se caracterizó mediante análisis termogravimétrico (TGA).

$H H V\left({ }^{M J} / \mathrm{kg}\right)=0.338 \mathrm{C}+1.428(H-O / 8)$

Donde $\mathrm{C}, \mathrm{H}, \mathrm{O}$, corresponden a las composiciones, en porcentaje, de cada elemento en el material.

\section{RESULTADOS Y DISCUSIÓN}

Los resultados se presentan divididos en: Preparación de la muestra, evaluación de las condiciones del proceso y caracterización del biocrudo.

\section{Preparación y caracterización de la materia prima}

Las muestras de Acacia mangium se homogenizaron mediante reducción del tamaño de partícula en un molino de cuchillas (0,8-1,4 mm). Se analizó la composición elemental de la madera de Acacia mangium, obteniéndose un contenido de carbono del $46,30 \%, 5,70 \%$ en hidrógeno, $46,65 \%$ en oxígeno, $1,32 \%$ en nitrógeno, valores similares a $48,9 \%$ C, 5,9\% hidrogeno, $44,5 \%$ oxígeno y $0,2 \%$ de nitrógeno, obtenidos por Minowa et al. La determinación del poder calorífico se llevó a cabo mediante el uso de la fórmula de Dulong, resultando en 15,46 MJ/Kg, valor levemente por debajo de 19,7MJ/Kg reportado por Minowa et al. (Minowa, 1998). Además, se determinó el contenido de cenizas con un $3,44 \%$ (por calcinación a $575^{\circ} \mathrm{C}$ ) y un contenido de azufre de $0,01 \%$.

\section{Evaluación de condiciones del proceso de licuefacción hidrotérmica de biomasa}

Con el fin de determinar el efecto de variables como el tiempo de reacción, la temperatura y la relación biomasa/solvente en el proceso de licuefacción hidrotérmica de Acacia mangium, se desarrolló el diseño experimental $3^{k}$, cuyas condiciones se muestran en la Tabla 2. Para esta etapa se utilizó como catalizador $\mathrm{K}_{2} \mathrm{CO}_{3}$ al $10 \%$ con respecto a la biomasa (Dimitriadis y Bezergianni, 2017). La Figura 2 muestra la distribución de productos obtenidos durante la licuefacción hidrotérmica de Acacia a las condiciones de la tabla 2, en base seca y libre de inorgánicos.

Los rendimientos más altos hacia biocrudo se obtuvieron a $270^{\circ} \mathrm{C}$, que fue la temperatura más baja evaluada. La formación de compuestos gaseosos aumenta con la temperatura de reacción, debido a que se favorecen sucesivas reacciones de descomposición de los bio-polímeros de la biomasa maderable acacia. Entre tanto, a una temperatura de $270^{\circ} \mathrm{C}$, se observa una disminución del rendimiento hacia la formación de la fase gaseosa con el aumento en la cantidad de solvente empleado en la reacción. A las temperaturas de 300 y $330^{\circ} \mathrm{C}$, la formación de gases es mayor a medida que incrementa el tiempo de residencia de la mezcla reaccionante en el reactor. También se observa, en general, que la formación de residuo sólido disminuye con el aumento de la temperatura y de la cantidad de solvente. Los aumentos y disminuciones de residuo sólido con el tiempo de residencia se deben a bajas conversiones del sólido y a la re-polimerización 
de compuestos que conllevan a la formación de bio-char. Por otra parte, la formación de compuestos solubles en agua es muy semejante para todos los experimentos realizados. Sin embargo, para la temperatura más alta evaluada $\left(330^{\circ} \mathrm{C}\right)$, se puede observar una leve disminución en el rendimiento hacia la formación de compuestos solubles en agua, que se generan por las múltiples reacciones sucesivas de degradación de celulosa, hemicelulosa y lignina.

Tabla 2: Diseño Experimental licuefacción hidrotérmica de biomasa.

\begin{tabular}{|c|c|c|c|}
\hline Experimento & Temperatura $\left({ }^{\circ} \mathrm{C}\right)$ & Relación líquido/sólido (g/g) & Tiempo (min) \\
\hline 2 & 270 & 10 & 0 \\
\hline 7 & 270 & 10 & 30 \\
\hline 13 & 270 & 10 & 60 \\
\hline 20 & 270 & 15 & 0 \\
\hline 16 & 270 & 15 & 30 \\
\hline 21 & 270 & 15 & 60 \\
\hline 25 & 270 & 20 & 0 \\
\hline 26 & 270 & 20 & 30 \\
\hline 9 & 270 & 20 & 60 \\
\hline 27 & 300 & 10 & 0 \\
\hline 5 & 300 & 10 & 30 \\
\hline 15 & 300 & 10 & 60 \\
\hline 1 & 300 & 15 & 0 \\
\hline 12 & 300 & 15 & 30 \\
\hline 19 & 300 & 15 & 60 \\
\hline 14 & 300 & 20 & 0 \\
\hline 22 & 300 & 20 & 30 \\
\hline 11 & 300 & 20 & 60 \\
\hline 3 & 330 & 10 & 0 \\
\hline 17 & 330 & 10 & 30 \\
\hline 24 & 330 & 10 & 60 \\
\hline 6 & 330 & 15 & 0 \\
\hline 4 & 330 & 15 & 30 \\
\hline 18 & 330 & 15 & 60 \\
\hline 10 & 330 & 20 & 0 \\
\hline 23 & 330 & 20 & 30 \\
\hline 8 & 330 & 20 & 60 \\
\hline
\end{tabular}

En general, puede observarse que a $270^{\circ} \mathrm{C}$, el rendimiento aumenta con el tiempo y con la disminución en la relación biomasa/solvente. A $300^{\circ} \mathrm{C}$, el comportamiento en cuanto al rendimiento del biocrudo con respecto al tiempo de reacción y la cantidad de solvente, es diferente para cada caso: para una relación biomasa solvente 1:10, el rendimiento disminuye con el tiempo de reacción, para una relación 1:15 aumenta con el tiempo y para la relación 1:20 no hay un efecto notable del tiempo. Para la mayor temperatura de reacción evaluada, se observa una disminución en el rendimiento con el tiempo de reacción para las tres relaciones biomasa/solvente evaluadas, esto debido a que con el incremento de la temperatura se favorecen las reacciones de descomposición que conllevan a la formación de compuestos que enriquecen los demás productos y subproductos de la reacción, principalmente la fase acuosa y la fase gaseosa.

El análisis estadístico de los resultados obtenidos para la licuefacción hidrotérmica de la madera de Acacia (no mostrado), reveló que la variable más influyente en el rendimiento a biocrudo son la temperatura y la combinación tiempo - temperatura; a mayor temperatura y menor tiempo, mayor rendimiento; por el contrario a menor temperatura y mayor tiempo, menor rendimiento; por lo cual, el máximo rendimiento a biocrudo con agua como solvente fue de $40,2 \%$ y se obtuvo a $270 \stackrel{\circ}{\circ}$, 60 minutos y una relación biomasa/solvente 1:20 (experimento 9). El orden de los rendimientos a biocrudo mas altos y sus condiciones 
de operación fue: $40,2 \%$ (exp. $9,270^{\circ} \mathrm{C}, \mathrm{L} / \mathrm{S}=20,60 \mathrm{~min}$ ), 38,4\% (exp. 19, 300 $\mathrm{C}, \mathrm{L} / \mathrm{S}=15,60 \mathrm{~min}$ ), 36,0\% (exp. $\left.6,330^{\circ} \mathrm{C}, \mathrm{L} / \mathrm{S}=15,0 \mathrm{~min}\right), 35,1 \%$ (exp. $7,270^{\circ} \mathrm{C}, \mathrm{L} / \mathrm{S}=10,30 \mathrm{~min}$ ).

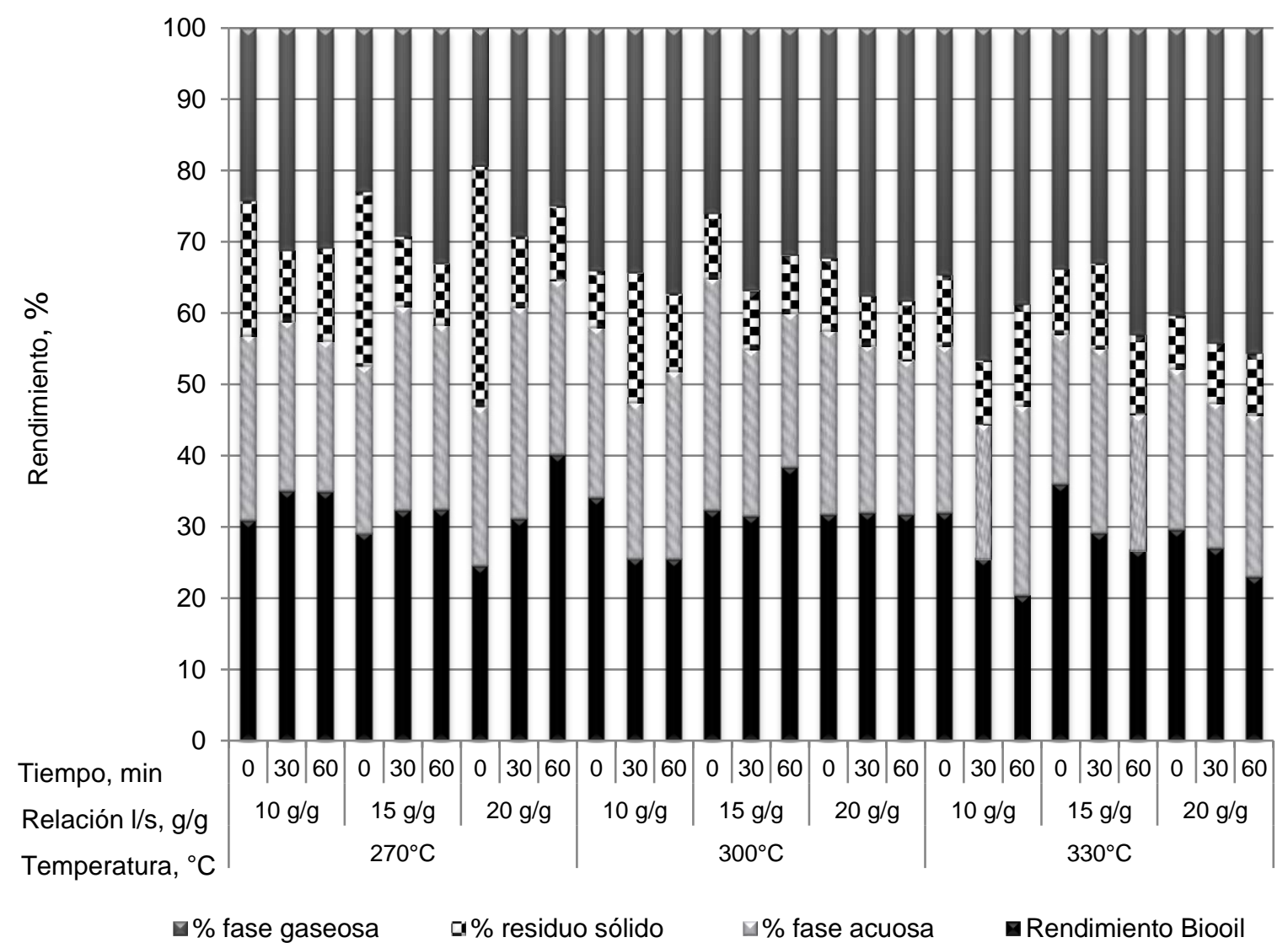

Fig. 2: Rendimientos de biocrudo (insoluble en agua), biocrudo soluble en agua, residuo sólido y productos gaseosos. La primera fila del eje $x$ es el tiempo en minutos, la segunda la relación biomasa/solvente y la tercera es la temperatura. Condiciones experimentales de la tabla 2.

Otros autores al realizar la licuefacción hidrotérmica de biomasas maderables obtuvieron a condiciones similares rendimiento de $34,9 \%$ a condiciones de $280{ }^{\circ} \mathrm{C}$ y 15 minutos de reacción con el catalizador de carbonato de potasio para la madera de cerezo (cherry) y para un ciprés a las mismas condiciones se obtuvo un rendimiento de $28,8 \%$ (Bhaskar et al. 2008), por otro lado, Kubilay Tekin obtuvo un rendimiento de madera (beech) a $300^{\circ} \mathrm{C}$ de $29,8 \%$ con un poder calorífico de $23,81 \mathrm{Mj} / \mathrm{kg}$.

Para licuefacción de biomasas maderables provenientes de la china se reportaron rendimientos entre 27.38 y $32.21 \%$ (Zhong y Wei 2004). Se ha reportado además que el rendimiento a partir de madera de pino en presencia de varios solventes (agua, acetona y etanol) incrementa cuando se incrementa la temperatura cerca de los $300^{\circ} \mathrm{C}$ para el agua y $400^{\circ} \mathrm{C}$ para la acetona y el etanol (Liu y Zhang 2008). Durante la licuefacción con agua de aserrín de pino se encontró que al disminuir la relación biomasa/solvente se incrementa el rendimiento a biocrudo y también la formación de residuos sólidos (Cheng et al. 2010).

En la tabla 3 se observa el análisis elemental realizado a cada uno de los biocrudos obtenidos en cada ensayo de la tabla 2 (licuefacción con agua y con catalizador), con el fin de determinar el poder calorífico según la fórmula de Dulong, la cual permite determinar cuál es la cantidad de energía contenida en cada kilogramo de biocrudo. El aumento en el poder calorífico logrado bajo las diferentes condiciones de reacción empleadas, implica polimerizaciones secundarias, generadas por reacciones de oxidación ocurridas entre los productos primarios de hidrólisis de los componentes. Se reportan los valores del poder calorífico en orden decreciente y a las condiciones experimentales de la tabla 2.

Con respecto a la composición elemental, se puede observar en general un aumento drástico en el contenido de carbono y una disminución significativa en el contenido de oxígeno con respecto a la materia prima inicial. 
Tabla 3: Composición elemental y poder calorífico del biocrudo

\begin{tabular}{|c|c|c|c|c|c|}
\hline Experimento & $\% N$ & $\% C$ & $\% H$ & $\% O$ & HHV (MJ/kg) \\
\hline 4 & 1,58 & 82,19 & 8,00 & 13,68 & 36,74 \\
\hline 19 & 1,44 & 72,98 & 7,08 & 14,78 & 32,13 \\
\hline 12 & 1,00 & 76,51 & 6,45 & 16,66 & 32,10 \\
\hline 17 & 2,78 & 75,52 & 6,41 & 14,43 & 32,10 \\
\hline 24 & 1,20 & 73,59 & 6,56 & 13,01 & 31,91 \\
\hline 6 & 1,12 & 75,05 & 6,55 & 16,94 & 31,69 \\
\hline 16 & 0,73 & 75,01 & 6,52 & 17,50 & 31,54 \\
\hline 18 & 1,69 & 72,29 & 6,74 & 14,58 & 31,45 \\
\hline 8 & 1,95 & 73,62 & 6,52 & 15,93 & 31,35 \\
\hline 5 & 2,37 & 72,70 & 6,73 & 18,02 & 30,97 \\
\hline 26 & 0,75 & 73,86 & 6,57 & 19,06 & 30,93 \\
\hline 10 & 1,01 & 72,87 & 6,45 & 16,69 & 30,86 \\
\hline 1 & 0,74 & 73,50 & 6,35 & 18,84 & 30,55 \\
\hline 23 & 1,37 & 71,84 & 6,34 & 15,68 & 30,52 \\
\hline 11 & 1,33 & 71,99 & 6,53 & 17,98 & 30,45 \\
\hline 15 & 0,98 & 72,51 & 6,33 & 17,50 & 30,42 \\
\hline 22 & 0,94 & 72,09 & 6,10 & 16,38 & 30,14 \\
\hline 27 & 1,27 & 70,77 & 6,34 & 18,13 & 29,73 \\
\hline 9 & 1,26 & 69,67 & 6,46 & 17,61 & 29,63 \\
\hline 2 & 0,30 & 72,46 & 6,07 & 19,88 & 29,62 \\
\hline 21 & 1,53 & 69,69 & 6,16 & 15,54 & 29,57 \\
\hline 14 & 1,80 & 70,02 & 6,52 & 19,84 & 29,43 \\
\hline 7 & 1,41 & 68,61 & 6,57 & 17,98 & 29,36 \\
\hline 20 & 1,84 & 67,36 & 6,31 & 19,05 & 28,38 \\
\hline 13 & 1,57 & 66,57 & 6,42 & 19,02 & 28,28 \\
\hline 3 & 0,18 & 66,45 & 5,98 & 17,04 & 27,95 \\
\hline 25 & 1,26 & 67,76 & 5,96 & 20,38 & 27,77 \\
\hline Blanco (Acacia) & 1,32 & 46,30 & 5,70 & 46,65 & 15,46 \\
\hline
\end{tabular}

El valor de poder calorífico más alto de $36,74 \mathrm{MJ} / \mathrm{kg}$ se obtuvo bajo condiciones de $330^{\circ} \mathrm{C}, 30$ minutos y una relación biomasa/solvente 1:15 (experimento 4). El análisis estadístico (no mostrado) permitió establecer (a partir de p-value) que las variables más influyentes sobre el poder calorífico del biocrudo son la temperatura y la interacción de tiempo-temperatura; también se observa que a mayores tiempos de reacción el poder calorífico aumenta. En la figura 3 se resumen los resultados de poder calorífico y rendimiento de los biocrudos obtenidos en los experimentos de la tabla 2. Estos valores son comparables con los reportados por otros autores para licuefacción de madera de acacia bajo condiciones similares. Minowa et al. llevó a cabo licuefacción de $10 \mathrm{~g}$ de madera de acacia (Acacia mangium) con $100 \mathrm{~mL}$ de agua destilada en presencia de $0,5 \mathrm{~g}$ de $\mathrm{NaCO}_{3}$ a $300^{\circ} \mathrm{C}$ por $30 \mathrm{~min}$, obteniendo un biocrudo con una composición elemental de $71,7 \%$ de carbono, $6,5 \%$ en hidrógeno, 0,4\% en nitrógeno, $21,5 \%$ en oxígeno.

El poder calorífico real reportado por el autor, $29,8 \mathrm{MJ} / \mathrm{Kg}$ es comparable con los más bajos hallados en presente diseño experimental (Minowa, 1998). Por otro lado, la madera de acacia (Acacia mangium Willd) también se ha usado como materia prima en otros procesos de termoconversión. Charusiri et al. Llevaron a cabo la pirólisis de esta madera observando el efecto de la temperatura entre 440 y $580^{\circ} \mathrm{C}$, tasa de alimentación de biomasa entre 0,3 y $0,9 \mathrm{Kg} / \mathrm{h}$ y tasa de flujo de nitrógeno entre 80 y $160 \mathrm{~cm}^{3} / \mathrm{min}$ en un reactor en continuo usando $1 \%$ de dolomita como catalizador. Los mejores resultados de rendimiento $(44,78 \%)$ y poder calorífico $(24,32 \mathrm{MJ} / \mathrm{Kg})$ fueron obtenidas a $540^{\circ} \mathrm{C}$, tasa de alimentación de $0,45 \mathrm{Kg} / \mathrm{h}$ y flujo de nitrógeno de $155 \mathrm{~cm}^{3} / \mathrm{min}$ con una carga de catalizador de dolomita de $1 \%$, resultados que, comparados con los de licuefacción muestran una desventaja en cuanto a la densificación energética y rendimiento de producto se refiere (Charusiri, 2017).

Se observa que el poder calorífico no presenta una amplia variación y oscila alrededor de $30 \mathrm{MJ} / \mathrm{kg}$. Por el contrario, el rendimiento a biocrudo si presenta una variación muy amplia entre 20 y $42 \%$. Ninguna de estas dos variables, poder calorífico y rendimiento a biocrudo, muestra una clara tendencia o correlación con las condiciones experimentales. Debido a que los valores de rendimiento a biocrudo y su poder calorífico no 
siguen una misma tendencia y esto dificulta la selección de las mejores condiciones de proceso, se calculó una nueva variable respuesta denominada Rendimiento Energético (RE), que es la cantidad de energía contenida en el biocrudo que se obtiene por cada $100 \mathrm{~kg}$ de biomasa alimentada; ésta se obtiene multiplicando el rendimiento a biocrudo por su poder calorífico. La figura 4 muestra la variación decreciente de RE en función del número del experimento, el cual a su vez se puede correlacionar con las condiciones experimentales de la tabla 2.

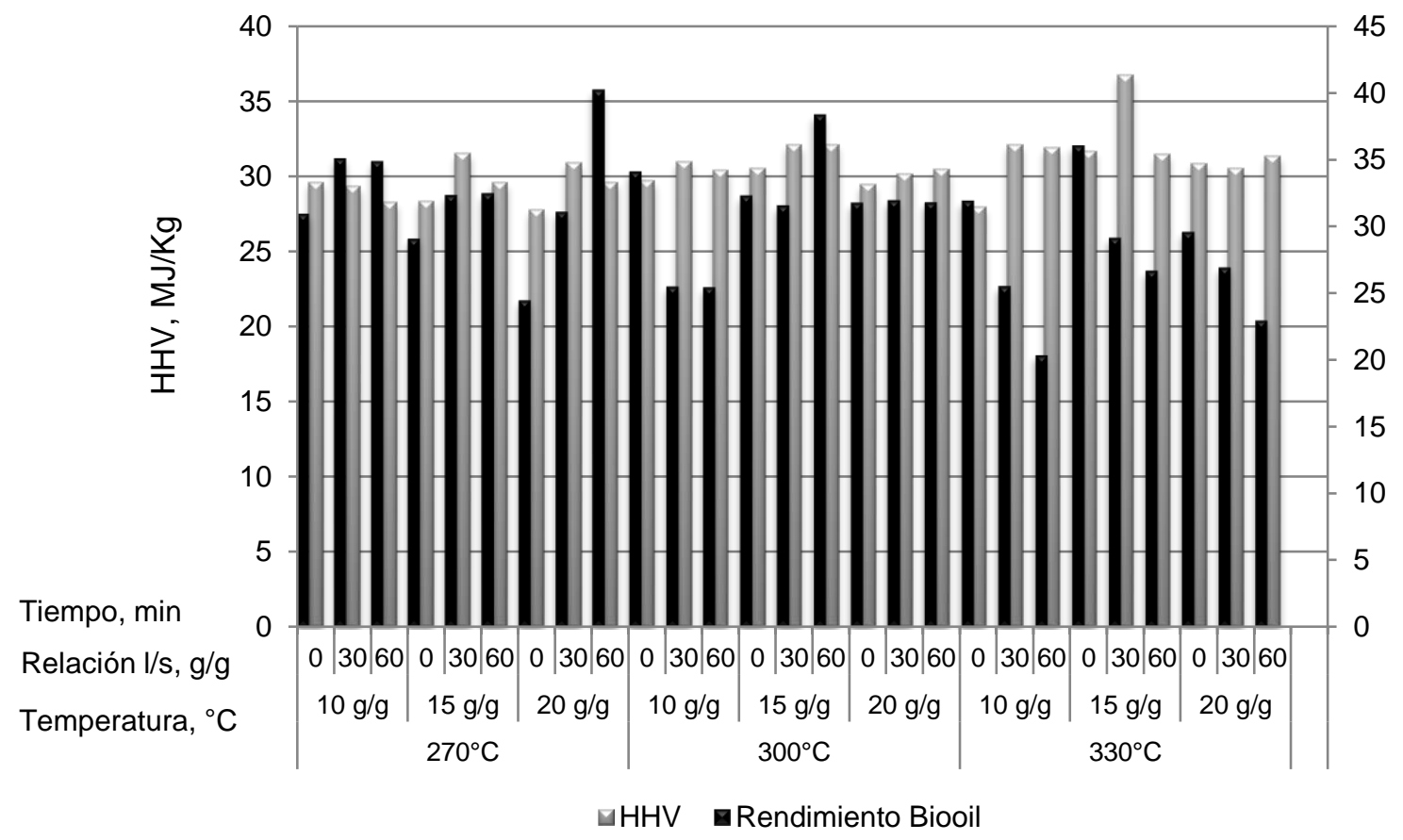

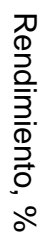

Fig. 3: Rendimiento a biocrudo y poder calorífico para la licuefacción hidrotérmica de Acacia. La primera fila del eje $x$ es el tiempo en minutos, la segunda la relación biomasa/solvente y la tercera es la temperatura. Condiciones experimentales de la tabla 2.

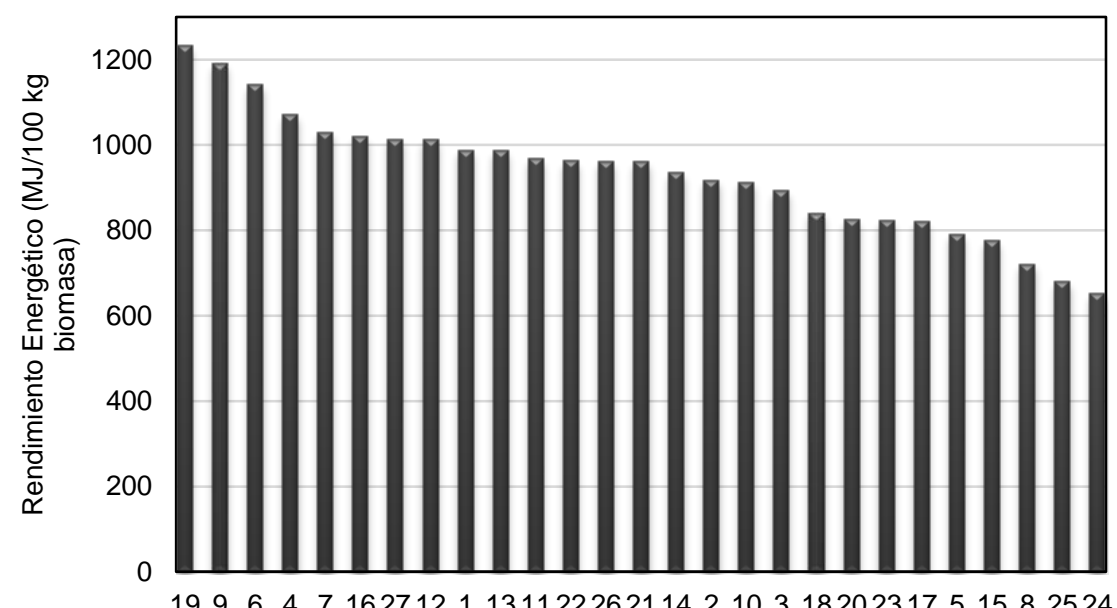

No. Experimento

Fig.4: Rendimiento energético RE según el número de experimento de la tabla 2.

El orden de los experimentos con mayor rendimiento energético (en orden decreciente) y sus respectivas condiciones de operación es: experimento $19\left(300^{\circ} \mathrm{C}, 60 \mathrm{~min}\right.$ y $\left.\mathrm{L} / \mathrm{S}=15\right)$, experimento $9\left(270^{\circ} \mathrm{C}, 60 \mathrm{~min}\right.$ y $\mathrm{L} / \mathrm{S}=20)$, experimento $6\left(330^{\circ} \mathrm{C}, 0 \mathrm{~min}\right.$ y $\left.\mathrm{L} / \mathrm{S}=15\right)$ y experimento $4\left(330^{\circ} \mathrm{C}, 30 \mathrm{~min}\right.$ y $\left.\mathrm{L} / \mathrm{S}=15\right)$. Sin embargo, hay que analizar que los experimentos 9 y 19 tienen tiempos de reacción de 60 minutos, que es un tiempo de residencia bastante prolongado que conduciría a equipos de mayor tamaño o a menor capacidad de la planta para un tamaño fijo. El experimento que les sigue, el 6, tiene el menor de los tiempos de residencia y la menor relación L/S entre estos cuatro experimentos, además, su rendimiento energético está bastante cerca al del experimento 19. Una relación L/S más baja conduce también a equipos de menor tamaño y a menor consumo energético. Por estas razones, se seleccionaron las condiciones del experimento 6 como las mejores para continuar el resto de la investigación. 


\section{Efecto de la carga del catalizador}

Se evaluó el efecto de la carga del catalizador $\left(\mathrm{K}_{2} \mathrm{CO}_{3}\right)$, pues se ha reportado que éste mejora la producción de biocrudo y reduce la cantidad de residuo sólido (Karagoz et al. 2005). Se establecieron 4 niveles de carga de catalizador: $0,5,10$ y $20 \%$ en peso con respecto a la biomasa alimentada, que se evaluaron a las mejores condiciones encontradas en la etapa anterior, las cuales son $330^{\circ} \mathrm{C}, 0$ minutos de reacción y una relación de 1:15 biomasa/solvente (experimento 6). En la figura 5 se muestra la distribución de productos para cada carga de catalizador empleada. El aumento en la carga de catalizador muestra un aumento en la formación de compuestos solubles en agua y la disminución en la formación de residuo sólido. El aumento en la carga del catalizador parece favorecer las reacciones que dan lugar a la formación de compuestos solubles en agua derivados de la degradación de celulosa, hemicelulosa y lignina, que llevan a la formación de ácidos orgánicos y derivados furánicos. (Akhtar et al., 2011).

Cuando la licuefacción hidrotérmica se debe sólo al efecto térmico ( $0 \%$ catalizador), los análisis GC-MS (no mostrados pues son tablas muy extensas), indican que los componentes predominantes en el biooil producido son provenientes de la degradación de la lignina y de los azúcares que componen la celulosa y hemicelulosa, llevando a la formación principalmente de 2,6 dimetoxi fenol, y ácido n-hexadecanoico, y se observó además que el $60 \%$ de las áreas corresponden a derivados fenólicos. Con la adición de catalizador, se observan tendencias diferentes en cuanto a la composición y proporción de los compuestos presentes en la fase biocrudo.

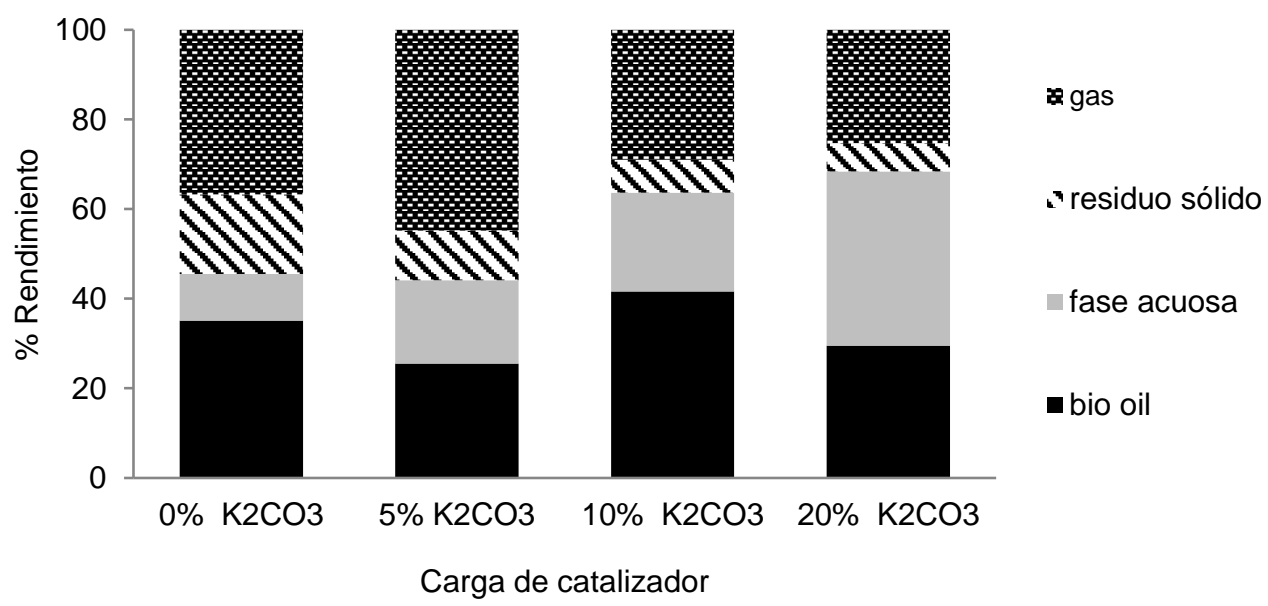

Fig. 5: Distribución de productos en la licuefacción hidrotérmica de acacia empleando distintas cargas de catalizador.

Se observa un aumento en las áreas de 2,6 dimetoxi fenol con el aumento en la carga de catalizador, además de otros compuestos como alcoholes, derivados de ácidos grasos, hidrocarburos alifáticos de cadena larga y compuestos aromáticos poli sustituidos. Cuando se emplearon cargas de 5,10 y $20 \%$ de carbonato de potasio, las proporciones en las áreas con respecto a la formación de compuestos fenólicos fueron 62, 57 y $72 \%$, respectivamente, y se observó que para las cargas de 5 y $10 \%$ hay más formación de hidrocarburos lineales de alto peso molecular y de ácidos grasos y sus derivados, que para $0 \%$ y $20 \%$ de carga de catalizador. Se evidencia además que para la mayor carga de catalizador hay una mayor formación de compuestos mono y poliaromáticos sustituidos.

A los biocrudos obtenidos con las diferentes cargas de catalizador se les realizó un análisis termogravimétrico con el fin de conocer la volatilidad de los productos obtenidos, los resultados se muestran en la tabla 4. De estos resultados se desprende que el mejor biocrudo se obtiene cuando se emplea el carbonato de potasio a un $10 \%$ con respecto a la biomasa, puesto que, además de tener el mayor rendimiento hacia el biocrudo, se obtiene el mayor poder calorífico y sus compuestos son más volátiles ya que cerca del $52 \%$ es volátil a $370^{\circ} \mathrm{C}$. Como referencia, a esta temperatura el diésel y el biodiesel volatilizan en un $83.6 \%$ y $78.8 \%$ respectivamente, lo que indica que al biocrudo obtenido le falta etapas de mejoramiento para ser utilizado como un combustible convencional. Comparado con el trabajo de Minowa et al., llevado a cabo a $300^{\circ} \mathrm{C}$ por 30 min con una carga de $5 \%$ de $\mathrm{NaCO}_{3}$ como catalizador $(29,8 \mathrm{MJ} / \mathrm{Kg})$, el obtenido en la presente investigación bajo una carga similar de catalizador resulto en un biocrudo con más bajo poder calorífico, 26,51 MJ/Kg (Minowa, 1998). 
Tabla 4: Distribución de puntos de ebullición, poder calorífico y rendimiento a diferentes cargas de catalizador.

\begin{tabular}{|c|c|c|c|c|c|c|c|}
\hline Carga de catalizador & $\begin{array}{c}<200 \stackrel{\circ}{\circ} \mathrm{C} \\
\%\end{array}$ & $\begin{array}{c}200-250 \stackrel{\circ}{ } \mathrm{C} \\
\%\end{array}$ & $\begin{array}{c}250-300 \stackrel{\circ}{\circ} \mathrm{C} \\
\%\end{array}$ & $\begin{array}{c}300-370{ }^{\circ} \mathrm{C} \\
\%\end{array}$ & $\begin{array}{c}>370^{\circ} \mathrm{C} \\
\%\end{array}$ & $\begin{array}{c}H H V \\
(M J / k g)\end{array}$ & $\begin{array}{c}\text { \%Rendimiento } \\
\text { a biocrudo }\end{array}$ \\
\hline $0 \% \mathrm{~K}_{2} \mathrm{CO}_{3}$ & 13,3 & 6,1 & 8,6 & 13,9 & 58,1 & 25,38 & 35,1 \\
\hline $5 \% \mathrm{~K}_{2} \mathrm{CO}_{3}$ & 13,7 & 7 & 8,7 & 14,4 & 56,2 & 26,51 & 25,5 \\
\hline $10 \% \mathrm{~K}_{2} \mathrm{CO}_{3}$ & 13,9 & 9,6 & 8,8 & 20,1 & 47,6 & 31,69 & 36,0 \\
\hline $20 \% \mathrm{~K}_{2} \mathrm{CO}_{3}$ & 8,4 & 8,3 & 8,5 & 15,4 & 59,4 & 28,12 & 29,6 \\
\hline
\end{tabular}

\section{Efecto del tipo de solvente}

En la figura 6 se muestra el efecto de diferentes solventes a las mejores condiciones encontradas con agua $\left(330^{\circ} \mathrm{C}, 0\right.$ minutos, $\mathrm{L} / \mathrm{S}=15$ y $\left.10 \% \mathrm{~K}_{2} \mathrm{CO}_{3}\right)$ en atmosfera de argón. Se ha reportado que la adición de solventes orgánicos como la acetona, el etanol, el fenol, entre otros, en los procesos de licuefacción mejora el rendimiento a biocrudo (Demirbas, 2008; Liu y Zhang, 2008).

Un resultado muy interesante es la licuefacción usando la acetona como solvente, ya que permite obtener el más alto rendimiento hacia la formación de biocrudo. Liu et al. (2008) estudiaron el efecto de solventes como el agua, etanol y acetona en la licuefacción de una madera de pino a condiciones similares de temperatura y presión y una relación de biomasa solvente de $1 / 6$; encontraron que a $400^{\circ} \mathrm{C}$ los mayores rendimientos fueron $26,5 \%$ con etanol, $18,6 \%$ con agua y $20 \%$ con acetona. Se puede establecer que efectivamente la licuefacción mediante etanol y acetona mejora la conversión hacia la formación de biocrudo, en este caso para la acacia a las condiciones evaluadas se observa que el mejor solvente es la acetona ya que permite obtener un rendimiento de biocrudo muy alto con respecto a la biomasa alimentada.

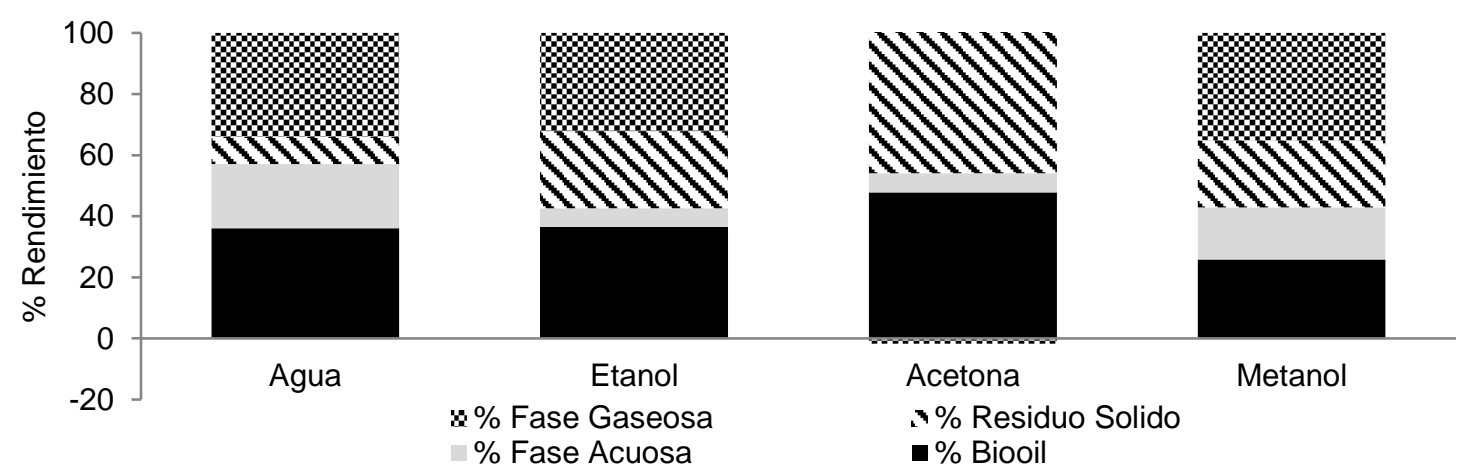

Fig. 6: Distribución de productos en la licuefacción de acacia con diferentes solventes.

En la tabla 5 se muestran los poderes caloríficos obtenidos mediante la fórmula de Dulong y la distribución de puntos de destilación para los biocrudos obtenidos a partir de los diferentes solventes. Las condiciones son las del experimento 6 , descritas en la tabla 2 . Se observa que el poder calorífico más alto se obtiene para el metanol, sin embargo, el rendimiento hacia la formación de biocrudo es muy bajo, menor de cuando se utiliza el agua. En cuanto al etanol, el poder calorífico está por debajo del obtenido con agua y el rendimiento a biocrudo es muy similar al obtenido con el agua. Con la acetona el resultado es muy interesante, ya que además de que presenta un mayor rendimiento hacia la formación de biocrudo, su poder calorífico es ligeramente mayor al de la licuefacción convencional con agua. En cuanto a los puntos de ebullición, la tabla 5 muestra que con la acetona se obtiene el biocrudo más liviano, seguido por el etanol, el metanol y por último el agua.

Tabla 5: Distribución de puntos de ebullición, poder calorífico y rendimiento la licuefacción solvotérmica de acacia mangium*

\begin{tabular}{|c|c|c|c|c|c|c|c|}
\hline \multirow{2}{*}{ Solvente } & \multicolumn{5}{|c|}{ Distribución puntos de ebullición (\%peso)) } & \multirow{2}{*}{$\begin{array}{c}H H V \\
(M J / k g)\end{array}$} & \multirow{2}{*}{$\begin{array}{c}\text { \%Rendimiento a } \\
\text { Biocrudo }\end{array}$} \\
\hline & $<200^{\circ} \mathrm{C}$ & $200-250^{\circ} \mathrm{C}$ & $250-300 \stackrel{\circ}{ } \mathrm{C}$ & $300-370^{\circ} \mathrm{C}$ & $>370^{\circ} \mathrm{C}$ & & \\
\hline Agua & 13,9 & 9,6 & 8,8 & 20,1 & 47,6 & 31,69 & 36,0 \\
\hline Etanol & 28,9 & 15 & 10,4 & 9,1 & 36,6 & 28,98 & 36,5 \\
\hline Metanol & 22,6 & 13,4 & 9,7 & 14,2 & 40,1 & 32,39 & 25,8 \\
\hline Acetona & 39,7 & 16,4 & 9,2 & 10,7 & 24 & 31,65 & 47,8 \\
\hline
\end{tabular}




\section{Efecto de la atmósfera reductora}

Con el fin de favorecer la hidrogenación de los combustibles obtenidos se realizó el mejor experimento con agua como solvente $330^{\circ} \mathrm{C}, 0$ min, una $\mathrm{L} / \mathrm{S}=15,10 \% \mathrm{~K}_{2} \mathrm{CO}_{3}$ (experimento 6), pero con una atmosfera de $\mathrm{H}_{2}$ a 10 bares de presión inicial. No se encontró diferencia significativa en las variables respuesta, el rendimiento pasó de 36 a 37,9\% y el poder calorífico de 31,69 a 32,54 MJ/kg, esto se debe a que en el análisis elemental se encontró menor contenido de oxígeno y mayor de hidrógeno. Adicional a esto, se obtuvo un combustible menos pesado evidenciado en el análisis termo gravimétrico (tabla 6 ). Por último, se evaluó la atmósfera reductora utilizando acetona como solvente y las mejores condiciones encontradas en la primera etapa (experimento 6). Con estas condiciones el rendimiento a biocrudo fue de $89,6 \%$ con respecto a la biomasa y su poder calorífico de $35,66 \mathrm{MJ} / \mathrm{Kg}$, esto corresponde a un rendimiento energético $(\mathrm{RE})$ de $3195 \mathrm{MJ} /(100 \mathrm{~kg}$ biomasa). Estos incrementos tan marcados en el rendimiento másico a biocrudo y en el rendimiento energético se explican por la remoción de oxígeno gracias a la hidrogenación parcial del biocrudo.

Tabla 6: Distribución de puntos de ebullición en atmósferas reductoras y no reductoras.

\begin{tabular}{cccccc}
\hline Atmosfera de reacción & $<200{ }^{\circ} \mathrm{C} \%$ & $200-250{ }^{\circ} \mathrm{C} \%$ & $250-300{ }^{\circ} \mathrm{C} \%$ & $300-370{ }^{\circ} \mathrm{C} \%$ & $>370{ }^{\circ} \mathrm{C} \%$ \\
\hline Argón & 13,9 & 9,6 & 8,8 & 20,1 & 47,6 \\
Hidrógeno & 25,2 & 13,9 & 15,4 & 26,4 & 19,1 \\
\hline
\end{tabular}

\section{CONCLUSIONES}

En la evaluación del proceso de licuefacción solvotérmica de madera de acacia realizada en este estudio, se puede concluir:

1) Que, a una temperatura de $270^{\circ} \mathrm{C}$, una relación solvente (agua)/biomasa de 20 y un tiempo de reacción de 60 minutos (experimento 9) se obtiene el más alto rendimiento hacia la formación de biocrudo $(40,2 \%)$, con un poder calorífico de 29,63 MJ/kg;

2) Con base en la nueva variable calculada Rendimiento Energético (RE) se encontró que las mejores condiciones de reacción son $330^{\circ} \mathrm{C}$, una relación solvente (agua)/biomasa de 15 y un tiempo de reacción de 0 minutos (experimento 6), con las que se obtiene un biocrudo con un rendimiento másico del $36 \%$ y un poder calorífico de $31,7 \mathrm{MJ} / \mathrm{kg}$;

3) Al evaluar diferentes cargas del catalizador $\mathrm{K}_{2} \mathrm{CO}_{3}(0,5,10$ y 20\%) se encontró que la mejor carga es el $10 \%$, pues con ella se obtuvo el biocrudo más liviano con el mayor rendimiento másico (36\%) y con el poder calorífico más alto $(31,69 \mathrm{MJ} / \mathrm{kg})$;

4) Al evaluar agua, metanol, etanol y acetona como solventes, se encontró que solo con acetona se obtienen resultados relevantes, pues el rendimiento a biocrudo se incrementó a 47,8 \%, su poder calorífico se mantuvo casi estable $(31,65 \mathrm{MJ} / \mathrm{kg}$ ) y el producto fue mucho más liviano que el obtenido con agua (y con los demás solventes);

5) Al evaluar la licuefacción convencional con agua bajo una atmosfera de hidrógeno no se encontraron diferencias significativas en las variables respuesta. 6) La atmósfera de hidrógeno sí mostró un fuerte efecto positivo en la licuefacción usando acetona como solvente, pues se presentó un aumento en el rendimiento a biocrudo y su poder calorífico, lo que indica un mayor grado de hidrogenación del producto.

\section{AGRADECIMIENTOS}

Los autores agradecen el apoyo económico para el desarrollo de esta investigación, enmarcada dentro del proyecto Captación de $\mathrm{CO}_{2}$ de industria para la producción de biocombustibles financiado por Ruta N, la Universidad de Antioquia y Argos S.A.

\section{REFERENCIAS}

Akhtar, J., y N. Amin, A review on process conditions for optimum bio-oil yield in hydrothermal liquefaction of biomass, Renewable and Sustainable Energy Reviews, 15(3), 1615-1624 (2011)

Anastasakis, K. y A. Ross, Hydrothermal liquefaction of the brown macro-alga Laminaria Saccharina: Effect of reaction conditions on product distribution and composition, Bioresource Technology, 102, 4876-4883 (2011)

Bhaskar, T., A. Sera, A. Muto e Y. Sakata, Hydrothermal upgrading of wood biomass: influence of the addition of $\mathrm{K}_{2} \mathrm{CO}_{3}$ and cellulose/lignin ratio, Fuel, 87, 10-11 (2008)

Charusiri, W. y N. Numcharoenpinij, Characterization of the optimal catalytic pyrolysis conditions for bio-oil production from brown salwood (Acacia mangium Willd) residues, Biomass and Bioenergy, 106, 127-136 (2017) 
Cheng, S. y otros cuatro autores, Highly efficient liquefaction of woody biomass in hot-compressed alcohol-water cosolvents, Energy and Fuels, 24(9), 4659-4667 (2010)

Demirbas, A., Production of biodiesel from algae oils, Energy Sources, 31,163-168 (2008)

Dimitriadis, A. y S. Bezergianni, Hydrothermal liquefaction of various biomass and waste feedstocks for biocrude production: A state of the art review, Renewable and Sustainable Energy Reviews, 68 (September 2016), 113-125 (2017)

Duan, P. y otros cinco autores, Hydrothermal processing of duckweed: Effect of reaction conditions on product distribution and composition, Bioresource Technology, 135, 710-719 (2012)

Duan, P. y otros siete autores, Thermo-chemical conversion of Chlorella pyrenoidosa to liquid biofuels, Bioresource technology, 133, 197-205 (2013)

Duan, P. y P. Savage, Hydrothermal Liquefaction of a Microalga with Heterogeneous Catalysts, Industrial \& Engineering Chemistry Research, 50(1), 52-61 (2011)

Espitia, M. y otros cuatro autores, Ganancia genética esperada en la selección de acacia (acacia mangium willd.) en Córdoba (Colombia), Rev. U.D.C.A Act. \& Div. Cient., 13 (2), 99-107 (2010)

Garcia-Perez, M. y otros cuatro autores, Characterization of bio-oils in chemical families, Biomass and Bioenergy, 31(4), 222-242 (2007)

Karagoz, S., T. Bhaskar, A. Muto e Y. Sakata, Comparative studies of oil compositions produced from sawdust, rice husk, lignin and cellulose by hydrothermal treatment, Fuel, 84(7-8), 875-884 (2005)

Kersten, S.R.A., D. Knežević. Y R.H. Venderbosch, Production of biofuels via hydrothermal conversion. In Handbook of Biofuels Production, 478-492 (2011)

Klass, D., Thermal Conversion: Pyrolysis and liquefaction, Biomass for renewable energy, fuels and chemicals, 651, (1998)

Liu, Z. y F.S. Zhang, Effects of various solvents on the liquefaction of biomass to produce fuels and chemical feedstocks, Energy Conversion and Management, 49(12),3498-3504 (2008)

Lloyd, T.A. y C.E. Wyman, Combined sugar yields for dilute sulfuric acid pretreatment of corn stover followed by enzymatic hydrolysis of the remaining solids, Bioresource Technology, 96 (18 SPEC. ISS.), 1967-1977 (2005)

Mazaheri, H., K.T. Lee y A.R. Mohamed, Influence of temperature on liquid products yield of oil palm shell via subcritical water liquefaction in the presence of alkali catalyst, Fuel Processing Technology, 110,197-205 (2013)

Minowa, T., T. Kondo y S.T.Sudirjo, Thermochemical liquefaction of Indonesian biomass residues, Biomass and Bioenergy, 14(5-6), 517-524 (1998)

Oasmaa, A., E. Kuoppala e Y. Solantausta, Fast Pyrolysis of Forestry Residue. 2. Physicochemical Composition of Product Liquid, Energy and Fuels, 17, 433-443 (2003)

Proexport, Sector Forestal en Colombia, Proexport Colombia, 1-17 (2012)

RIA, Reforestadora Integral de Antioquia, Informe gestión 2013 reforestadora integral de antioquia s.a., Medellín (2013)

Tekin, K., S. Karagoz y S. Bektas, Hydrothermal liquefaction of beech wood using a natural calcium borate mineral, Journal of Supercritical Fluids, 72, 134-139 (2012)

UPME / Ministerio de Minas y Energía / República de Colombia, Atlas del Potencial Energético de la Biomasa Residual en Colombia (2010)

Vardon, D.R. y otros cuatro autores, Thermochemical conversion of raw and defatted algal biomass via hydrothermal liquefaction and slow pyrolysis, Bioresource Technology, 109, 178-187 (2012)

Xu, C. y J. Lancaster, Conversion of secondary pulp/paper sludge powder to liquid oil products for energy recovery by direct liquefaction in hot-compressed water; Water research, 42(6-7), 1571-1582 (2008)

Yang, X. y otros cinco autores, Selective extraction of bio oil from hydrothermal liquefaction of Salix psammophila by organic solvents with different polarities through multistep extraction separation, BioResources, 9(3), 5219-5233 (2014)

Yuan, X.Z. y otros cuatro autores, Comparative Studies of Products Obtained at Different Temperatures during Straw Liquefaction by Hot Compressed Water, Energy \& Fuels, 23(6), 3262-3267 (2009)

Zhang, J. y otros cuatro autores, Hydrothermal liquefaction of Chlorella pyrenoidosa in sub- and supercritical ethanol with heterogeneous catalysts, Bioresource technology, 133, 389-397 (2013)

Zhong, C. y X. Wei, A comparative experimental study on the liquefaction of wood, Energy, 29(11), 1731-1741 (2004)

Zhou, D. y otros cuatro autores, Hydrothermal liquefaction of macroalgae Enteromorpha prolifera to Bio-oil, Energy Fuels, 24(7), 4054-4061 (2010)

Zhuang, Y. y otros cinco autores, Microwave-assisted direct liquefaction of Ulva prolifera for bio-oil production by acid catalysis, Bioresource technology, 116,133-139 (2012)

Zou, X. y otros cinco autores, Mechanisms and Main Regularities of Biomass Liquefaction with Alcoholic Solvents, Energy Fuels, 23(10), 5213-5218 (2009) 